\title{
Application of NGS in Diagnosis of Tuberculous Pleurisy with Multiple Negative Tests: A Case Report
}

\author{
Honglin $\mathrm{Wu}^{1,2}$ \\ Jiahui Wei ${ }^{1}$ \\ Dujuan $\mathrm{Yu}^{\prime}$ \\ 'Department of Respiratory Medicine, \\ China-Japan Union Hospital of Jilin \\ University, Changchun I30033, People's \\ Republic of China; ${ }^{2}$ Department of \\ Gastroenterology, China-Japan Union \\ Hospital of Jilin University, Changchun \\ 130033, People's Republic of China
}

Correspondence: Jiahui Wei Department of Respiratory Medicine, China-Japan Union Hospital of Jilin University, No. 126, Xiantai Street, Nanguan District, Changchun City, Jilin

Province, People's Republic of China

$\mathrm{Tel} / \mathrm{Fax}+86-43 \mathrm{I}-84995860$

Email weijiahui@jlu.edu.cn
This article was published in the following Dove Press journal: Infection and Drug Resistance

Background: Tuberculous pleurisy is inflammation caused by direct infection of Mycobacterium tuberculosis (MTB) and/or delayed allergic reaction of the pleura to MTB thallus components. The diagnosis of tuberculous pleurisy is mainly confirmed by bacterial culture, smear staining or histopathology, but has some clinical limitations. Next-generation sequencing (NGS), as a new diagnostic technology, has good application prospects in the diagnosis of tuberculous pleurisy.

Case Presentation: A patient admitted with right pleural effusion and pneumonia was actively treated with anti-infection, anti-inflammatory and symptomatic support while various etiological tests of right pleural effusion were improved. However, all the etiological tests for MTB infection were negative. At this time, the patient's condition worsened and pleural effusion also appeared on the left side. In order to clarify the cause of the disease as soon as possible and prevent the disease from worsening again, the left and right pleural effusions of the patient were sent for NGS testing. The test results suggested MTB infection, which finally clarified the diagnosis of tuberculous pleurisy, and the next treatment plan of the patient was timely adjusted.

Conclusion: NGS is instructive in the diagnosis of tuberculous pleurisy when various conventional tests and imaging methods fail.

Keywords: tuberculous pleurisy, Mycobacterium tuberculosis, NGS, infection, pleural effusion

\section{Introduction}

Tuberculous pleurisy is caused by MTB infection, and some patients present with cough, fever, chest pain and even dyspnea, as well as a small to moderate amount of pleural effusion; a few patients may have a large amount of pleural fluid. The diagnosis of tuberculous pleurisy was mainly confirmed by pleural effusion culture and smear staining, or by pathological examination of pleural tissue samples. However, the low sensitivity of pleural fluid culture and smear staining, as well as the various limitations of pathological biopsy, make thediagnosis of tuberculous pleurisy more difficult in the clinical practice. NGS is a brand-new detection technology applied to the diagnosis of infectious diseases. The development of NGS provides an accurate and efficient method for understanding MTB infection at the genome-wide level, and provides a new perspective and method for the diagnosis and research of tuberculous pleurisy. 


\section{Case Presentation}

A 64-year-old, Asian male presented to the Department of Respiratory Medicine at our hospital (a tertiary care hospital) on May 16, 2020 for cough and sputum with dyspnea for six months. Before the patient's visit, he had gradually aggravated dyspnea and presented chest tightness, chest pain, shortness of breath, edema of lower limbs and other symptoms, which had affected his daily life. However, he did not go to the local hospital for treatment. The patient reported a 5-year history of hypertension, up to 220/ $180 \mathrm{mmHg}$, self-administered antihypertensive medication, and self-reported blood pressure control acceptable. He also had a 5-year history of previous cerebral infarction. No other history of disease has been reported. Physical examination showed a temperature of $36.4 \mathrm{C}$, apulse of 119 beats/ min, a breath of 20 beats/min, and ablood pressure of 153/ $129 \mathrm{mmHg}$. Sporadic wet rales can be heard in both lungs, no obvious abnormal heart sounds in the heart, and multiple enlarged lymph nodes can be touched in the neck, axilla and inguinal regions. The results of chest computed tomography (CT) (Day -2) with our hospital showed (Figure1 A1 and A2): right interlobar fissure, reticular nodular, diffuse thickening; right pleural effusion with partial package, scattered inflammation in the middle and lower lobes of the right lung with local external pressure atelectasis, scattered alveoli in both lungs; diffuse small nodular high density shadow in
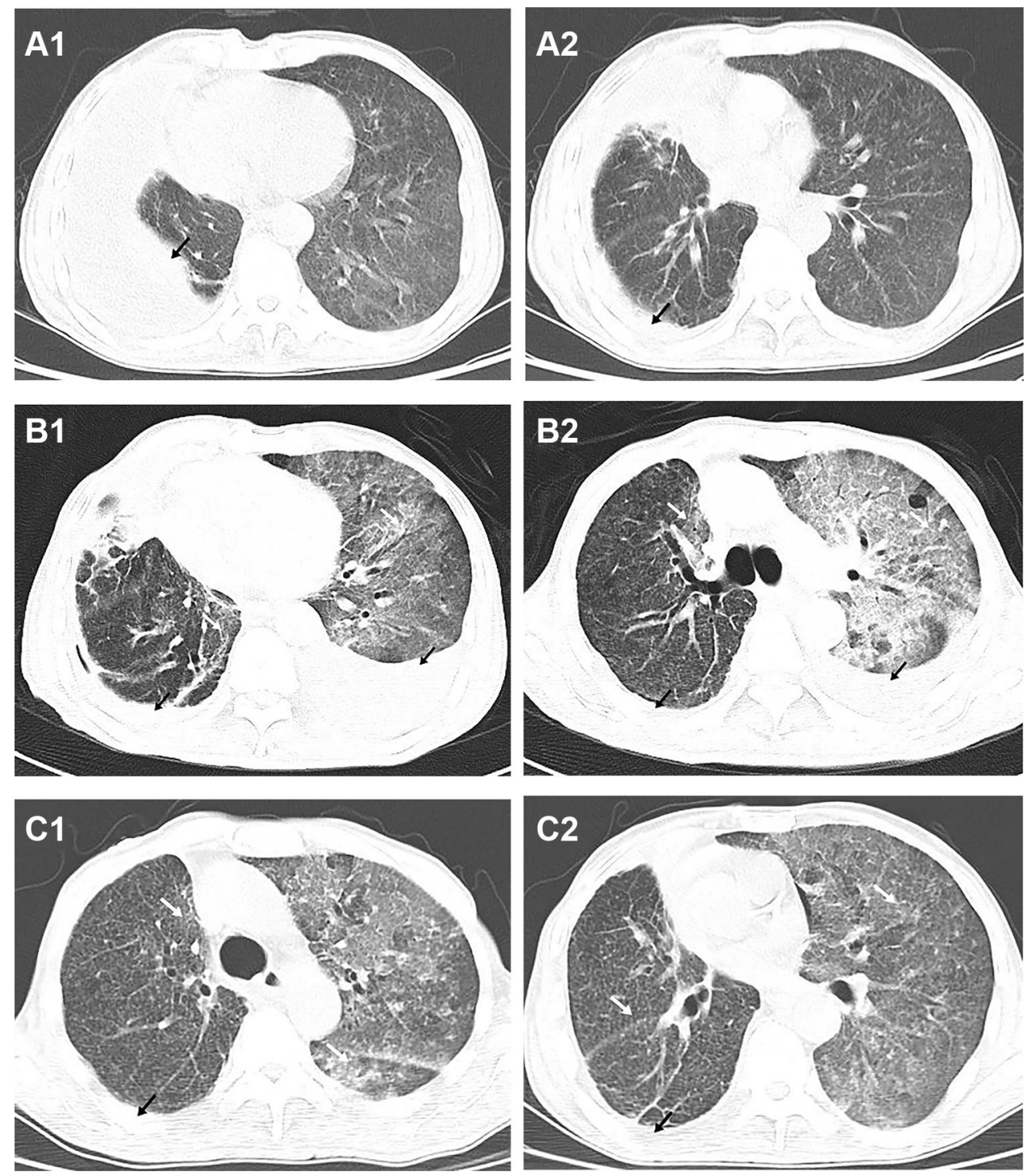

Figure I Comparison of cross-sectional chest CT images at different time points. Before admission (Day -2 ), a large pleural effusion (black arrow) was seen in the right lung on two cross sections (Al and A2); When the patient's condition worsened (Day 10), new pleural effusion (black arrow) in the left lung and diffuse patchy shadow (white arrow) in both lungs were shown in two cross sections (B I and B2); Before discharge (Day I5), two cross-sections of chest CT (CI and C2) showed absorption of left pleural effusion, reduction of right pleural effusion (black arrow), and reduction of diffuse patchy shadow in both lungs (white arrow). 
both lungs; plain strip shadow in the lower lingual segment of the upper lobe of the left lung. Laboratory examination results after admission: white blood cell $4.6 \times 10^{9} / \mathrm{L}$, neutrophil $\% 88.0 \%$, lymphocyte $0.19 \times 10^{9} / \mathrm{L}$, eosinophils 0.00 x $10^{9} / \mathrm{L}$, procalcitonin $0.27 \mathrm{ng} / \mathrm{mL}, \mathrm{N}$-terminal probrain natriuretic peptide (NT-proBNP) $295.00 \mathrm{pg} / \mathrm{mL}$, D-dimer (D-D) $3.42 \mu \mathrm{g} / \mathrm{mL}$, total protein (TP) $55.03 \mathrm{~g} / \mathrm{L}$, albumin (ALB) $27.81 \mathrm{~g} / \mathrm{L}$, no bacterial growth in blood culture (liquid culture), sputum culture (solid culture) for Streptococcus viridis (upper respiratory tract colonization bacteria). The detection of antibodies against Mycoplasma pneumoniae and Chlamydia pneumoniae showed that Chlamydia pneumoniae immunoglobulin G (IgG) 130.0 $\mathrm{AU} / \mathrm{mL}$, the rest of the antibodies were within the normal range, the respiratory pathogen spectrum antibody immunoglobulin $\mathrm{M}(\mathrm{IgM})$ test was negative, and the rest of the blood collection results were basically normal or abnormal results had no obvious clinical significance. Thoracic color Doppler ultrasound showed a $6.2 \mathrm{~cm}$ dark fluid area in the right thoracic cavity, and cardiac ultrasound results showed aortic sclerosis, normal left ventricular systolic function measurements, and tachycardia. Based on the current findings, the patient was initially diagnosed with 1) right pneumonia, 2) right pleural effusion, 3) double pulmonary bullae, 4) hypertension. After initial diagnosis, the patient was given symptomatic and supportive treatment such as anti-infection, anti-inflammation, cough relief and phlegm elimination, blood pressure lowering and diuresis.

Refer to the diagnosis process of adult unilateral pleural effusion diagnosis guide, ${ }^{1}$ we consider further clarifying the etiology of pleural effusion in this patient, and performing puncture and drainage of right pleural effusion and submitting it for examination. The test results (Table 1) classify the nature of pleural effusion according to Light criteria, ${ }^{1}$ and finally determine the exudative pleural effusion. Thereafter, relevant examinations were further improved to clarify the cause of exudative pleural effusion in this patient. Among them, immune-related tests: the results of anti-nuclear antibody series (17items) were negative, the results of anti-neutrophil cytoplasmic antibody (ANCA) series were negative, the results of anticyclic citrullinated peptide (CCP) antibody were 19.43 $\mathrm{RU} / \mathrm{mL}$, the results of immunoglobulin + complement + rheumatism suggested that complement C3 $0.73 \mathrm{~g} / \mathrm{L}, \mathrm{C}-$ reactive protein $186.34 \mathrm{mg} / \mathrm{L}$, all kinds of immune serological indicators were basically normal, only the slight decrease of complement $\mathrm{C} 3$, while the significant increase of C-reactive protein was considered to be associated with pulmonary inflammation, so it was not considered as immune-related pleural effusion. Fungi-related detection: serum galactomannan test (galactomannan) $<0.25$, $1.3-$ beta-D glucan test $53.14 \mathrm{pg} / \mathrm{mL}$ (reference result: $<70$ $\mathrm{pg} / \mathrm{mL}$ indicates a lower risk of infection, 70-90 pg/mL is recommended for continuous monitoring, and $>95 \mathrm{pg} /$ $\mathrm{mL}$ is highly suspicious of infection), and pleural effusion related to fungal infection was not considered according to the test results. Thyroid function test: among the five items of thyroid function, only triiodothyronine T3 was slightly reduced by $0.95 \mathrm{nmol} / \mathrm{L}$, and the rest had no abnormalities, so pleural effusion caused by thyroid function changes was not considered. However, the interferon- $\gamma$ release assay (TSPOT) of tuberculosis infection was negative (-), and the multi-tumor markers were generally normal (among which the neuron-specific enolase was only slightly elevated,

Table I Pleural Effusion Test Results

\begin{tabular}{|l|l|l|l|l|}
\hline Pleural Effusion & Right Results & Left Results & Unit & Normal Range \\
\hline Color & Yellow & Yellow & - & - \\
The Levant test & Positive $(+)$ & Positive $(+)$ & - & - \\
Leukocyte count & 299 & 287 & $\times 10^{6} / \mathrm{L}$ & - \\
Sugar & 5.0 & 6.0 & $\mathrm{mmol} / \mathrm{L}$ & - \\
TP & 31.8 & 32.8 & $\mathrm{~g} / \mathrm{L}$ & - \\
Chloride & 99.2 & 100.4 & $\mathrm{mmol} / \mathrm{L}$ & - \\
LDH & 246.29 & 419.09 & $\mathrm{IU} / \mathrm{L}$ & $<248.00$ \\
Multiple puncture fluid culture (solid culture) & No bacteria cultured & No bacteria cultured & - & - \\
Acid-fast staining (multiple times) & No acid-fast bacilli & No acid-fast bacilli & - & - \\
ADA & 20.27 & 15.21 & $\mathrm{U} / \mathrm{L}$ & $0.00-40.00$ \\
Exfoliated cells from puncture fluid (multiple times) & No atypical cells & No atypical cells & - & - \\
CEA & I.4 & 4.7 & $\mathrm{ng} / \mathrm{mL}$ \\
\hline
\end{tabular}

Abbreviations: TP, total protein; LDH, lactate dehydrogenase; ADA, adenosine deaminase; CEA, carcinoembryonic antigen. 
without reference significance). The two most common causes of pleural effusion are tuberculosis and tumors. ${ }^{1}$ Although current tests for tuberculosis and tumors were negative, the possibility of tumors or tuberculosis could not be ruled out. Therefore, further detection methods were needed to clarify the etiology of pleural effusion.

Unfortunately, on the 8th day of admission, the patient's sudden dyspnea worsened, and NT-proBNP $2990 \mu \mathrm{pg} / \mathrm{mL}$ and D-D $3.42 \mu \mathrm{g} / \mathrm{mL}$ were reexamined. The patient was transferred to intensify care unit (ICU) for treatment. After that, no venous thrombosis was found by venous ultrasound of lower limbs, no pulmonary embolism was found by computed tomography angiography (CTA) of the chest. Chest CT (Day 10) showed (Figure1 B1 and B2): decreased right pleural effusion, increased left pleural effusion, increased diffuse patchy and grid lesions of both lungs (considering the possibility of pulmonary edema combined with inflammation). Based on the above findings, we excluded the aggravation of acute dyspnea caused by pulmonary embolism. Considering that this aggravation may be due to acute heart failure and pulmonary edema induced by pulmonary infection, the symptomatic symptoms of dyspnea gradually improved after full antiinfection, recombinant human brain natriuretic peptide anti-heart failure, diuresis and other supportive treatment. However, the cause of the pleural effusion was still not clear at this time, and thus the additional left pleural effusion was sent for puncture and drainage (Table 1), and then the left and right pleural effusions were sent to NGS for testing respectively at this time.

A total of 39,575,303 reads were obtained from right pleural fluid samples, and 68 Mycobacterium tuberculosis complexes were detected (Figure 2A and B). A total of $38,603,770$ reads were generated from left pleural fluid samples, and 1 read of Mycobacterium tuberculosis complex were detected (Figure 2C and D), covering $0.0010 \%$ $(43 / 4,370,115)$ of the references genome. In order to validate the results of NGS detection, the detected specimens were also tested by real-time quantitative polymerase chain reaction (qPCR). The results of qPCR showed that Mycobacterium tuberculosis complex was positive in the right pleural fluid specimen, while no MTB was detected in the left pleural fluid specimen.
A
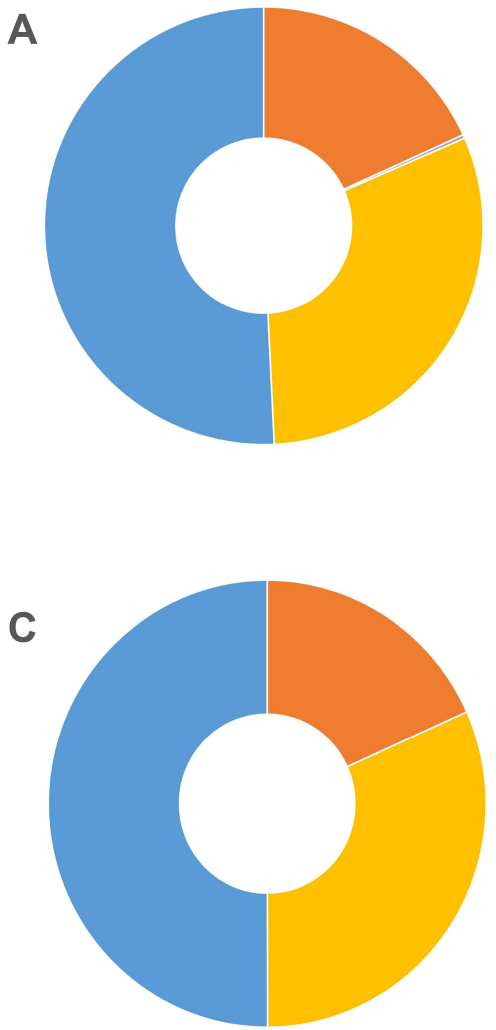

Microbial reads (140232) - bacteria $(25483,18.17 \%)$ - viruses $(377,0.27 \%)$ - fungi $(43179,30.79 \%)$ - parasites $(71193,50.77 \%)$

B

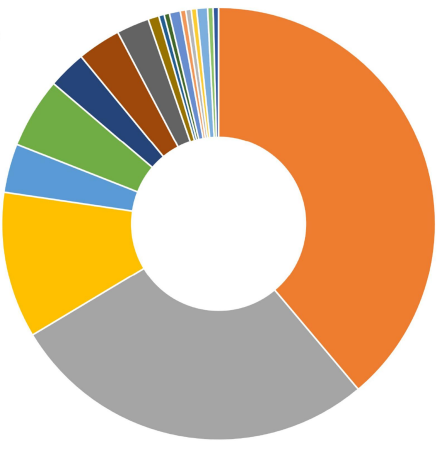

Bacterial reads on genus level (247) - Ralstonia (96, 38.87\%) Mycobacterium tuberculosis complex $(68,27.53 \%)$ - Burkholderia (27, 10.93\%) - Acinetobacter $(9,3.64 \%)$ - Bradyrhizobium $(13,5.26 \%)$ - Cutibacterium (7, 2.83\%) - Staphylococcus $(8,3.24 \%)$ - Sphingomonas $(6,2.43 \%)$ - Acidovorax $(2,0.81 \%)$ - Chryseobacterium $(1,0.40 \%)$ - Chryseobacterium $(1,0.40 \%)$ - Dermabacter $(1,0.40 \%)$ - Lactobacilus $(2,0.81 \%)$ Methylophilus $(1,0.40 \%)$ Methyloversatilis $(1,0.40 \%)$ Microbacterium $(1,0.40 \%)$ - Phycicoccus $(2,0.81 \%$ Proteus $(1,0.40 \%)$ - Xylanimonas $(1,0.40 \%)$
Microbial reads (154629) - bacteria $(28242,18.26 \%)$ - viruses $(3,0.00 \%)$ - fungi $(49019,31.70 \%)$ - parasites (77365, 50.03\%)
D

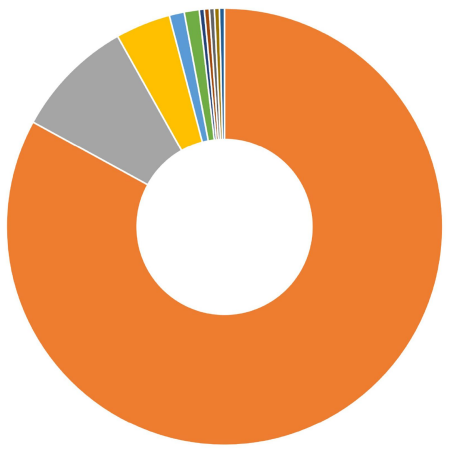

Bacterial reads on genus level (270) - Ralstonia $(244,90.37 \%)$

Bradyrhizobium (24, 8.89\%) I Sphingomonas (11, 4.07\%) - Acinetobacter (3, 1.11\%) - Cutibacterium (3, 1.11\%) - Corynebacterium $(1,0.37 \%)$ - Micrococcus (1, 0.37\%) - Mycobacterium tuberculosis complex (1, 0.37\%) - Sphingobium (1, 0.37\%) - Streptococcus (1, 0.37\%)

Figure 2 Taxonomic classification of sequencing readings read from pleural effusion. (A) Microbe sequences distribution in hydrothorax sample of right chest (B) Bacterium sequences distribution in hydrothorax sample of right chest on genus level (C) Microbe sequences distribution in hydrothorax sample of left chest (D) Bacterium sequences distribution in hydrothorax sample of left chest on genus level. 
MTB was detected in both NGS specimens, and MTB infection was confirmed in qPCR of right pleural effusion specimen, so the patient was diagnosed as tuberculous pleurisy. At this time, after full anti-infection, anti-inflammation, cough relief, phlegm resolving, symptomatic support treatment, the patient's symptoms of cough, expectoration, dyspnea and other symptoms have been better than those at admission, and the condition was relatively stable. Chest CT (Day 15) reexamination showed (Figure1 $\mathrm{C} 1$ and $\mathrm{C} 2$ ): absorption of left pleural effusion, reduction of right pleural effusion, improvement of bilateral pulmonary inflammation. According to the relevant national laws and regulations, tuberculosis is an infectious disease, and patients should receive designated treatment in infectious disease hospitals. In order to standardize anti-tuberculosis treatment and to prevent recurrence of the disease, the patient was discharged from the hospital and transferred to the tuberculosis infectious disease hospital for systematic treatment.

The patient's condition improved after standard antituberculosis treatment in Tuberculosis Infectious Disease Hospital, and the anti-tuberculosis treatment was effective, which confirmed the diagnosis of tuberculous pleurisy again. At this time, the patient was generally in good condition, and we contacted the patient to ask him to return for CT scan efficacy evaluation, but he just rejected the request and has not returned so far.

\section{Discussion and Conclusion}

Tuberculosis is a chronic infectious disease caused by MTB infection. There are at least nearly 10 million tuberculosis cases in the world and nearly 1.5 million people died of tuberculosis, so tuberculosis is still one of the most serious public health problems in the world. ${ }^{2}$ Tuberculosis accounts for about $80 \%$ of the total cases of tuberculosis, while the other $20 \%$ are extrapulmonary tuberculosis. The organs can be infected include lymph nodes, pleura, intestine, meninges, joints, bones, kidneys and skin. ${ }^{3}$ Tuberculous pleurisy is the second most common extrapulmonary tuberculosis after lymph node tuberculosis, accounting for about $4 \%$ of tuberculosis in the United States and Brazil and about $20 \%$ in South Africa. ${ }^{4}$ Tuberculous pleurisy has a higher disability rate than general pulmonary tuberculosis, ${ }^{5}$ so early diagnosis and treatment are crucial to the prognosis of the disease and the prevention and control of tuberculosis.

So far, the "gold standard" for the diagnosis of tuberculous pleurisy is still to find MTB from smear and culture of pleural effusion specimens, or the pathology of thoracic tissue specimen confirmed caseous granuloma and positive acid-fast staining. ${ }^{4,6}$ However, the positive rates of pleural effusion culture and acid-fast staining for tuberculous pleurisy are lower, ${ }^{7}$ and the culture time is longer. Therefore, the cultivation of pleural effusion and acidfast staining cannot meet the needs of clinical diagnosis of tuberculous pleurisy. Although pleural histology can be used for diagnosis, this diagnostic method cannot be fully implemented for intolerant elderly, potential complications and high-risk groups. Therefore, more new diagnostic techniques for tuberculous pleurisy need to be explored by clinical researchers.

NGS is an emerging high-throughput sequencing technology that uses genomics to sequence all pathogens in asample. The NGS detection methods used in this case, such as DNA extraction, library construction and sequencing procedures, are similar to those in published literatures. ${ }^{8,9}$ In brief, $0.5-3 \mathrm{~mL}$ hydrothorax sample from patient was collected according to standard procedures. $0.5 \mathrm{~mL}$ hydrothorax sample was added to $1.5 \mathrm{~mL}$ microcentrifuge tubes with $1 \mathrm{~g} 0.5 \mathrm{~mm}$ glass bead. The tubes were attached to a horizontal platform on a vortex mixer and agitated vigorously at $2800-3200 \mathrm{RPM}$ for $30 \mathrm{~min}$. $0.3 \mathrm{~mL}$ homogenized sample was separated into a new $1.5 \mathrm{~mL}$ microcentrifuge tube for DNA extraction using TIANamp Micro DNA Kit (DP316, TIANGEN BIOTECH) according to the manufacturer's recommendation. Then, DNA libraries were constructed through DNAfragmentation, end-repair, adapter-ligation and PCR amplification. Agilent 2100 was used for quality control of the DNA libraries. Quality qualified libraries were sequenced by BGISEQ-50 platform with single-end 50bp strategy. ${ }^{10}$ High-quality sequencing data were calculated by removing low-quality reads and then subtracting the human host sequence of the human reference genome (hg19) using Burrows-Wheeler alignment. ${ }^{11}$ After the removal of low-complexity reads, the remaining data were classified by simultaneously aligning to Microbial Genome Databases, consisting of 4945 whole genome sequence of viral taxa, 6350 bacterial genomes or scaffolds, 1064 fungi related to human infection, and 234 parasites associated with human diseases. The classification reference databases were downloaded from NCBI (ftp://ftp.ncbi.nlm.nih.gov/genomes/).

By using NGS for pathogen detection, specimens do not need to be cultured prior to detection, avoiding missed detection of difficult-to-culture pathogens. In addition, 
compared with traditional methods such as specific polymerase chain reaction (PCR), NGS detection does not need to presuppose specific pathogens and selected detection range. NGS can directly and nonspecifically determine all nucleic acid fragments to obtain genomic information of all microorganisms in samples, ${ }^{12}$ which avoids missed detection to the greatest extent. The application of qPCR in the left pleural effusion of this patient failed to detect MTB, possibly because the sequence of MTB amplified by qPCR was not the sequence of Mycobacterium tuberculosis complex detected by NGS, so specific detection could not be carried out. This also illustrates the possible limitations of qPCR detection and does not negate the accuracy of NGS detection results.

Recent studies have shown that NGS has been used for the diagnosis of various infectious diseases such as bacteria, ${ }^{13-15}$ fungi, ${ }^{16}$ mycoplasma, ${ }^{17}$ chlamydia, ${ }^{18}$ viruses, ${ }^{19-21}$ and parasites. ${ }^{22}$ These attempts suggest that NGS can be used as an alternative diagnostic or complementary method to help clinicians make appropriate decisions. Currently, NGS research on MTB has been used for epidemiological investigation, diagnosis of tuberculosis, gene polymorphism and drug resistance detection. ${ }^{23}$ Rapid diagnosis and drug resistance detection directly from sputum samples or alveolar lavage fluid is the ultimate application of NGS for tuberculosis. However, the main challenge of NGS application in clinical diagnosis lies in whether the microbial sequences tested conform to the etiological hypothesis and whether the results have clinical significance, because NGS detection is aimed at all nucleotide sequences in the specimen, which contain contaminants, normal colonizing microorganisms and true pathogenic microorganisms. ${ }^{13}$ The upper respiratory tract communicates with the outside world, there are many foreign microorganisms, and the oral cavity also contains many colonizing bacteria symbiotic with the human body. Therefore, the application of NGS to detect MTB in sputum or alveolar lavage fluid samples may have some limitations. The normal human pleural cavity is sterile. When the pleural effusion caused by pathogenic microorganisms occurs in the human body, the pathogen results obtained by using pleural effusion as NGS test specimens are more helpful for clinicians to make a judgment on the actual pathogen causing the disease. NGS is not currently the best choice for identification of MTB. However, for tuberculosis in which multiple traditional diagnostic methods have failed, NGS has become increasingly important as a test for MTB and is considered an important adjunct diagnostic tool.

In this case, the disease diagnosis process of this case is shown in Figure 3. The patient was admitted to the hospital with right pleural effusion, which was confirmed as exudative pleural effusion by biochemical testing. Since then, various etiological tests have been carried out for exudative pleural effusion. Among them, the tests for MTB, including T-SPOT, culture of pleural fluid, and antacid staining of pleural fluid were all negative, while chest CT showed severe pneumonia, no signs related to

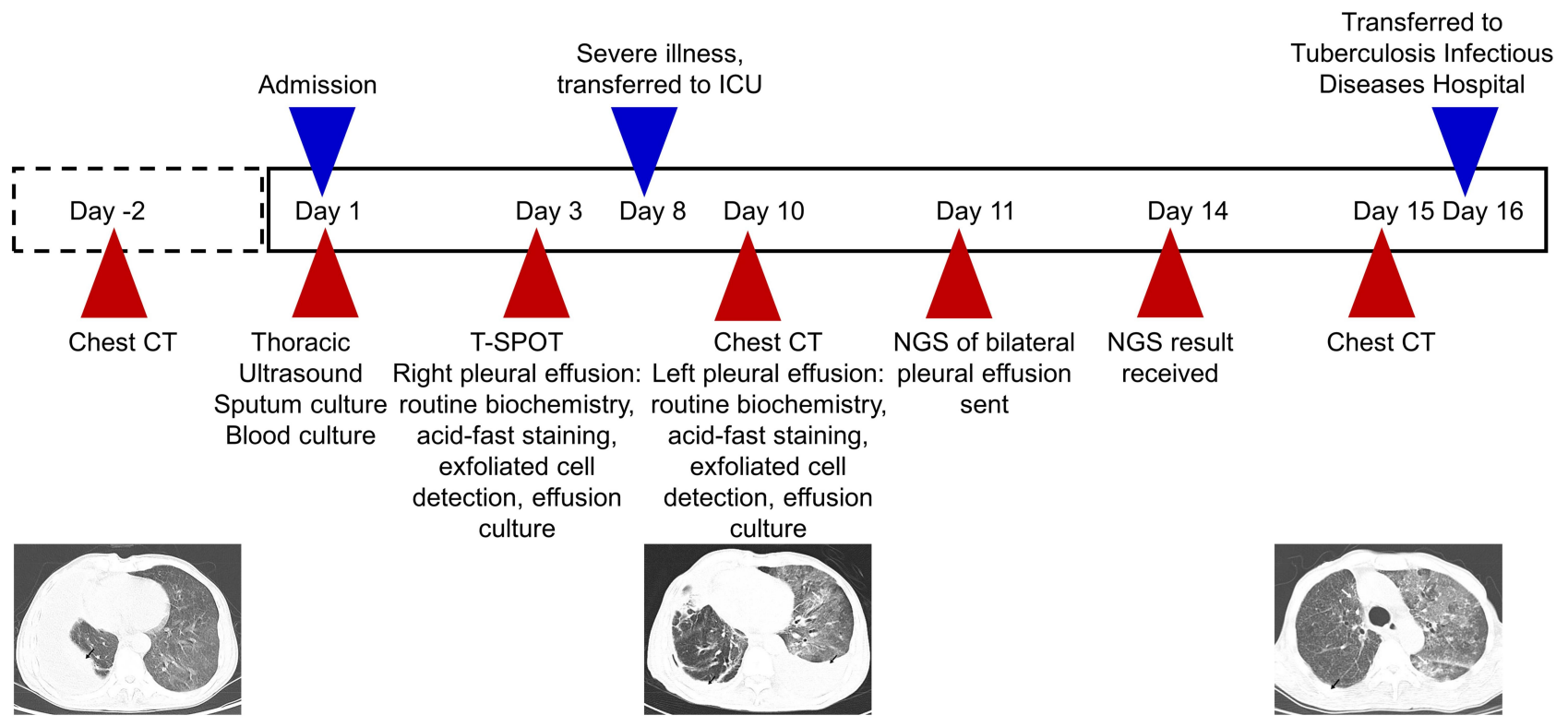

Figure 3 Patient diagnosis flow chart. 
tuberculosis infection. In the course of treatment, the condition worsened and pleural effusion began to appear on the left side. In order to clarify the cause as soon as possible and prevent the condition from worsening again, the left and right sides of the pleural effusion were sent to NGS for testing respectively. Both sides of NGS test results suggested MTB infection, PCR test also confirmed this. After this patient was treated with standardized antituberculosis treatment in the tuberculosis infectious disease hospital, his condition improved and was discharged. This treatment result also confirmed the diagnosis of tuberculosis pleurisy in this case.

This case report highlights the feasibility of using pleural effusion as an NGS test specimen in the diagnosis of tuberculous pleurisy. At the same time, NGS can help clinicians provide new diagnostic ideas for pathogenic infections that have failed various traditional diagnostic methods and further assist clinical decisionmaking.

\section{Abbreviations}

NGS, next-generation sequencing technology; PCR, polymerase chain reaction; qPCR, real-time quantitative polymerase chain reaction; MTB, Mycobacterium tuberculosis; T-SPOT, interferon- $\gamma$ release assay; NT proBNP, N-terminal probrain natriuretic peptide; ICU, intensify care unit; CTA, computed tomography angiography; CT, computed tomography; D-D, D-dimer; LDH, lactate dehydrogenase; TBIL, total bilirubin; DBIL, direct bilirubin; TP, total protein; ALB, albumin; ADA, adenosine deaminase; CEA, carcinoembryonic antigen.

\section{Consent}

Written informed consent was obtained from the patient for publication of this report. Detection of MTB is part of routine hospital laboratory procedures, so details of cases can be published without agency approval.

\section{Acknowledgments}

Thanks to BGI-Shenzhen Company for its technical and data support, Dr. Yang Zhou for reanalyzing and revising the method and results of mNGS analysis, and reviewing and approving the final version for submission.

\section{Author Contributions}

All authors made a significant contribution to the work reported, whether that is in the conception, study design, execution, acquisition of data, analysis and interpretation, or in all these areas; took part in drafting, revising or critically reviewing the article; gave final approval of the version to be published; have agreed on the journal to which the article has been submitted; and agree to be accountable for all aspects of the work.

\section{Disclosure}

The authors report no conflicts of interest in this work.

\section{References}

1. Hooper C, Lee YC, Maskell N, Group BTSPG. Investigation of a unilateral pleural effusion in adults: british Thoracic Society Pleural Disease Guideline 2010. Thorax. 2010;65(Suppl 2):ii4-ii17. doi:10.1136/thx.2010.136978

2. Jeanes C, O'Grady J. Diagnosing tuberculosis in the 21st century - Dawn of a genomics revolution? Int J Mycobacteriol. 2016;5(4):384-391.

3. Acharya B, Acharya A, Gautam S, et al. Advances in diagnosis of Tuberculosis: an update into molecular diagnosis of Mycobacterium tuberculosis. Mol Biol Rep. 2020;47(5):4065-4075. doi:10.1007/ s11033-020-05413-7

4. Light RW. Update on tuberculous pleural effusion. Respirology. 2010;15(3):451-458

5. Liu YC, Shin-Jung Lee S, Chen YS, Tu HZ, Chen BC, Huang TS. Differential diagnosis of tuberculous and malignant pleurisy using pleural fluid adenosine deaminase and interferon gamma in Taiwan. $J$ Microbiol Immunol Infect. 2011;44(2):88-94. doi:10.1016/j. jmii.2010.04.001

6. Diacon AH, Van de Wal BW, Wyser C, et al. Diagnostic tools in tuberculous pleurisy: a direct comparative study. Eur Respir J. 2003;22(4):589-591. doi:10.1183/09031936.03.00017103a

7. Anie Y, Sumi S, Varghese P, Madhavi LG, Sathish M, Radhakrishnan VV. Diagnostic approaches in patients with tuberculous pleural effusion. Diagn Microbiol Infect Dis. 2007;59(4):389-394. doi:10.1016/j. diagmicrobio.2007.06.022

8. Zhang HC, Ai JW, Cui P, et al. Incremental value of metagenomic next generation sequencing for the diagnosis of suspected focal infection in adults. $J$ Infect. 2019;79(5):419-425. doi:10.1016/j. jinf.2019.08.012

9. Miao Q, Ma Y, Wang Q, et al. Microbiological Diagnostic Performance of Metagenomic Next-generation Sequencing When Applied to Clinical Practice. Clin Infect Dis. 2018;67(suppl_2): S231-S240. doi:10.1093/cid/ciy693

10. Jeon YJ, Zhou Y, Li Y, et al. The feasibility study of non-invasive fetal trisomy 18 and 21 detection with semiconductor sequencing platform. PLoS One. 2014;9(10):e110240. doi:10.1371/journal.pone.0110240

11. Li H, Durbin R. Fast and accurate short read alignment with Burrows-Wheeler transform. Bioinformatics. 2009;25(14):17541760. doi:10.1093/bioinformatics/btp324

12. Jacob HJ. Next-generation sequencing for clinical diagnostics. $N$ Engl J Med. 2013;369(16):1557-1558. doi:10.1056/NEJMe1310846

13. Xu A, Zhu H, Gao B, et al. Diagnosis of severe community-acquired pneumonia caused by Acinetobacter baumannii through next-generation sequencing: a case report. BMC Infect Dis. 2020;20(1):45. doi:10.1186/s12879-019-4733-5

14. Jin W, Miao Q, Wang M, et al. A rare case of adrenal gland abscess due to anaerobes detected by metagenomic next-generation sequencing. Ann Transl Med. 2020;8(5):247. doi:10.21037/atm.2020.01.123

15. Huang Y, Ma Y, Miao Q, et al. Arthritis caused by Legionella micdadei and Staphylococcus aureus: metagenomic next-generation sequencing provides a rapid and accurate access to diagnosis and surveillance. Ann Transl Med. 2019;7(20):589. doi:10.21037/ atm.2019.09.81 
16. He BC, Liu LL, Chen BL, Zhang F, Su X. The application of nextgeneration sequencing in diagnosing invasive pulmonary aspergillosis: three case reports. Am J Transl Res. 2019;11(4):2532-2539.

17. Xiao N, Gai W, Hu WG, Li JX, Zhang Y, Zhao XY. Next-generationsequencing technology used for the detection of Mycoplasma hominis in renal cyst fluid: a case report. Infect Drug Resist. 2019;12:1073-1079. doi:10.2147/IDR.S198678

18. Zhang H, Zhan D, Chen D, et al. Next-generation sequencing diagnosis of severe pneumonia from fulminant psittacosis with multiple organ failure: a case report and literature review. Ann Transl Med. 2020;8(6):401. doi:10.21037/atm.2020.03.17

19. Fang $M$, Weng $X$, Chen L, et al. Fulminant central nervous system varicella-zoster virus infection unexpectedly diagnosed by metagenomic next-generation sequencing in an HIV-infected patient: a case report. BMC Infect Dis. 2020;20(1):1. doi:10.1186/s12879-020-4872-8
20. Zhang Y, Hong K, Zou Y, Bu H. Rapid detection of human herpes virus by next-generation sequencing in a patient with encephalitis. Virol J. 2019;16(1):104. doi:10.1186/s12985-019-1205-x

21. Wollants E, Smolders D, Naesens R, et al. Use of Next-Generation Sequencing for Diagnosis of West Nile Virus Infection in Patient Returning to Belgium from Hungary. Emerg Infect Dis. 2018;24 (12):2380-2382. doi:10.3201/eid2412.180494

22. Xie M, Zhou Z, Guo S, Li Z, Zhao H, Deng J. Next-generation sequencing specifies Angiostrongylus eosinophilic meningoencephalitis in infants: two case reports. Medicine. 2019;98(35):e16985. doi:10.1097/MD.0000000000016985

23. Comas I, Gil A. [Next generation sequencing for the diagnostics and epidemiology of tuberculosis]. Enferm Infecc Microbiol Clin. 2016;34(Suppl 3):32-39. doi:10.1016/S0213-005X(16)30217-8. Spanish.

\section{Publish your work in this journal}

Infection and Drug Resistance is an international, peer-reviewed openaccess journal that focuses on the optimal treatment of infection (bacterial, fungal and viral) and the development and institution of preventive strategies to minimize the development and spread of resistance. The journal is specifically concerned with the epidemiology of antibiotic resistance and the mechanisms of resistance development and diffusion in both hospitals and the community. The manuscript management system is completely online and includes a very quick and fair peerreview system, which is all easy to use. Visit http://www.dovepress.com/ testimonials.php to read real quotes from published authors. 